\title{
CHARACTERIZATIONS OF THE ESSENTIAL SPECTRUM OF F. E. BROWDER
}

\author{
BY DAVID LAY
}

Communicated by Felix Browder, August 16, 1967

Let $T$ be a densely defined closed linear operator on a Banach space $X$. F. E. Browder [1] has defined the essential spectrum of $T$, $\operatorname{ess}(T)$, to be the set of complex numbers $\lambda$ such that at least one of the following conditions is satisfied:

(i) The range $R(\lambda-T)$ of the operator $\lambda-T$ is not closed in $X$;

(ii) $\mathrm{U}_{k \geq 0} \Re\left[(\lambda-T)^{k}\right]$ is of infinite dimension, $(\Re(S)$ being the null space of the operator $S$ );

(iii) The point $\lambda$ is a limit point of the spectrum of $T$.

In [7], M. Schechter discusses two other sets of complex numbers, $\sigma_{e w}(T)$ and $\sigma_{e m}(T)$, which have also been called the essential spectrum of $T$ (cf. [10]). He characterizes $\sigma_{e m}(T)$ as the largest subset of the spectrum of $T$ which remains invariant under compact perturbations of $T$. Although $\sigma_{e m}(T)$ is in general a proper subset of ess $(T)$, Schechter gives conditions which guarantee that ess $(T)$ will remain invariant under compact (and certain other) perturbations of $T$. The proofs of these results usually reduce to showing that $\sigma_{e m}(T)=\operatorname{ess}(T)$.

In this paper we replace Schechter's conditions on $T$ by a condition on the perturbing operator and show that ess $(T)$ is invariant under compact (and certain other) perturbations of $T$, provided the perturbing operators commute with $T$. We shall say that a linear operator $C$ commutes with $T$ if (i) the domain of $C, D(C)$, contains the domain of $T$, (ii) $C x \in D(T)$ whenever $x \in D(T)$, (iii) and $T C x=C T x$ for $x \in \mathscr{D}\left(T^{2}\right)$.

Following the notation and terminology of [9], we denote the dimension of the null space or nullity of an operator $S$ by $n(S)$ and the codimension of the range or defect of $S$ by $d(S)$. The ascent of $S, \alpha(S)$, is the smallest in teger $p$ such that $\mathfrak{N}\left(S^{p}\right)=\mathfrak{N}\left(S^{p+1}\right)$, and the descent of $S, \delta(S)$, is the smallest integer $q$ such that $R\left(S^{q}\right)=R\left(S^{q+1}\right)$. (It may happen that $\alpha(S)=\infty$ or $\delta(S)=\infty$.) Suppose that $\lambda_{0}$ is a pole of order $p$ of the resolvent operator $(\lambda-T)^{-1}$ and let $E$ be the spectral projection corresponding to the spectral set $\left\{\lambda_{0}\right\}$. The range of $E$ is the null space of $\left(\lambda_{0}-T\right)^{p}$ and the dimension of this space is called the rank of the pole $\lambda_{0}$.

TheOREM 1. Let $T$ be a densely defined closed linear operator on a 
Banach space $X$. Let $\lambda_{0}$ be a point of the spectrum of $T$. The following statements are equivalent:

(1) $\lambda_{0}$ is not in $\operatorname{ess}(T)$.

(2) $\lambda_{0}$ is a pole of the resolvent $(\lambda-T)^{-1}$ of finite rank.

(3) $\lambda_{0}$ has finite ascent, descent, and defect.

(4) $n\left(\lambda_{0}-T\right)=d\left(\lambda_{0}-T\right)<\infty$ and $\alpha\left(\lambda_{0}-T\right)<\infty$.

The equivalence of (1) and (2) was proved by F. E. Browder in Lemma 17 of [1]. Since that time results of Kaashoek [3], Taylor [9] and the author [6] have provided tools for giving a short proof of the equivalence of (1), (2), and (4) without requiring that $T$ have a dense domain.

Theorem 2. Let $T$ be a closed linear operator on a Banach space $X$. $A$ point $\lambda_{0}$ in the spectrum of $T$ is a pole of the resolvent of finite rank if and only if there is a compact linear operator $C$ with $C(D(T)) C D(T)$ and $T C x=C T x$ for $x \in D\left(T^{2}\right)$ such that $\lambda_{0}-(T+C)$ has a bounded inverse defined on all of $X$.

Corollary. Let $T$ be a closed linear operator on a Banach space $X$. Then $\operatorname{ess}(T)$ is the largest subset of the spectrum $\sigma(T)$ which remains invariant under perturbations of $T$ by compact operators which commute with $T$, i.e.

$$
\begin{gathered}
\operatorname{ess}(T)=\{\lambda \mid \lambda \in \sigma(T+C) \text { for every compact operator } C \text { such that } \\
\left.C(D(T)) \subset D(T) \text { and } T C x=C T x \text { for } x \in D\left(T^{2}\right)\right\} .
\end{gathered}
$$

Both $\sigma_{e m}(T)$ and ess $(T)$ are also invariant under certain unbounded perturbations. Suppose that $T$ is a closed linear operator in $X$ and $C$ is a linear operator with $D(C) \supset D(T)$. We say that $C$ is $T$-closable if $x_{n} \rightarrow 0, T x_{n} \rightarrow 0, C x_{n} \rightarrow z$ for $\left\{x_{n}\right\} \subset D(T)$ implies $z=0$. The operator $C$ is $T$-compact if for any sequence $\left\{x_{n}\right\} \subset D(T)$ satisfying

$$
\left\|x_{n}\right\|+\left\|T x_{n}\right\| \leqq \text { const. }
$$

the sequence $\left\{C x_{n}\right\}$ has a convergent subsequence. The operator $C$ is $T$-pseudo-compact if for any sequence $\left\{x_{n}\right\} \subset D(T)$ satisfying

$$
\left\|x_{n}\right\|+\left\|T x_{n}\right\|+\left\|C x_{n}\right\| \leqq \text { const. }
$$

the sequence $\left\{C x_{n}\right\}$ has a convergent subsequence. Theorem 3 below is the analogue of Theorems 2.1 and 2.2 of [7] (although in that paper $T$ and $C$ were densely defined).

THEOREM 3. Let $T$ be a closed linear operator on a Banach space $X$. Then $\operatorname{ess}(T)$ is the largest subset of the spectrum of $T$ which remains in- 
variant under perturbations of $T$ by operators $C$ which commute with $T$ and are either T-compact or are T-closable and T-pseudo-compact.

The compactness condition on the perturbing operator $C$ may be generalized in another direction. It is well known that

$$
n(T)-d(T)=n(T+C)-d(T+C)
$$

when $C$ is a strictly singular operator (or is strictly singular relative to $T$, cf. [4]), or $C$ is an inessential operator (cf. [5]). From Schechter's characterization [7] of $\sigma_{e m}(T)$ as the complement in the complex plane of the set of points $\lambda$ for which $n(\lambda-T)=d(\lambda-T)<\infty$ it follows immediately that $\sigma_{e m}(T)$ is the largest subset of the spectrum which remains invariant under perturbations of $T$ by strictly singular or inessential operators. Even more is true in the analogous situation for $\operatorname{ess}(T)$. The ideal of strictly singular operators and the ideal of inessential operators are both contained in a set of bounded linear operator called Riesz operators [2].

A Riesz operator $R$ is characterized by the property that it is a bounded linear operator with $d(\lambda-R)<\infty$ for all $\lambda \neq 0$ [6].

THEOREM 4. Let $T$ be a closed linear operator on a Banach space $X$. Then ess $(T)$ is the largest subset of the spectrum of $T$ which remains invariant under perturbations of $T$ by Riesz operators $R$ which commute with $T$.

Proofs of these results will appear elsewhere.

\section{REFERENCES}

1. F. E. Browder, On the spectral theory of elliptic differential operators. I, Math. Ann. 142 (1961), 22-130.

2. S. R. Caradus, Operators of Riesz type, Pacific J. Math. 18 (1966), 61-71.

3. M. A. Kaashoek, Ascent, descent, nullity and defect, a note on a paper by A. E. Taylor, Math. Ann. 172 (1967), 105-115.

4. T. Kato, Perturbation theory for nullity, deficiency and other quantities of linear operators, J. Analyse Math. 6 (1958), 261-322.

5. D. Kleinecke, Almost-finite, compact and inessential operators, Proc. Amer. Math. Soc. 14 (1963), 863-868.

6. D. C. Lay, Studies in spectral theory using ascent, descent, nullity and defect, Doctoral dissertation, University of California, Los Angeles, 1966.

7. M. Schechter, On the essential spectrum of an arbitrary operator. I, J. Math. Anal. Appl. 13 (1966), 205-215.

8. A. E. Taylor, Introduction to functional analysis, Wiley and Sons, New York, 1958.

9. - Theorems on ascent, descent, nullity and defect of linear operators, Math. Ann. 163 (1966), 18-49.

10. F. Wolf, On the essential spectrum of partial differential boundary problems, Comm. Pure Appl. Math. 12 (1959), 211-228.

University of California, Los Angeles and

UNIVERSITY OF MARYLAND 